\title{
Fish Bone Foreign Body: The Role of Imaging
}

\author{
Ayala Klein ${ }^{1}$ Sharon Ovnat-Tamir ${ }^{1} \quad$ Tal Marom $^{1} \quad$ Ofer Gluck $^{1} \quad$ Naomi Rabinovics ${ }^{1}$ Shay Shemesh ${ }^{1}$ \\ 1 Department of Otolaryngology-Head and Neck Surgery, Assuta \\ Ashdod University Hospital, Ashdod, Israel \\ Int Arch Otorhinolaryngol 2019;23:110-115.

\begin{abstract}
Address for correspondence Ayala Klein, MD, Department of Otolaryngology-Head and Neck Surgery, Assuta Ashdod University Hospital, 7 Ha'refua street, Ashdod, 7747629, Israel (e-mail: ayalakl@assuta.co.il; ayala.klain@gmail.com).
\end{abstract}

\begin{abstract}
Introduction Fish bone foreign body (FFB) impaction in the upper aerodigestive tract is a common cause for emergency department referral. Its management varies in both diagnosis and treatment paradigms. Fish bone foreign bodies are more commonly found in the oropharynx in cases of patients $<40$ years old, and in the esophagus in cases of patients $>40$ years old. Symptoms are typically non-indicative for the location of the FFB, with the exception of foreign body sensation at/superior to the cervical esophagus. A lack of findings during the physical examination is routinely followed by imaging, with computed tomography $(\mathrm{CT})$ being the preferred modality. In practice, many patients undergo unnecessary imaging studies, including CT scans.

Objectives To identify patients with suspected fish bone impaction who do not require $C T$ imaging and can be safely discharged.

Data Synthesis We have searched the PubMed database for the following medical subject headings (MeSH) terms: fish bone, fish foreign body AND oropharynx, hypopharynx, esophagus, flexible esophagoscopy, and rigid esophagoscopy. Our search in the

\section{Keywords}

- oropharynx

- hypopharynx

- esophagus

- flexible esophagoscopy

- foreign bodies

- bone and bones

English language yielded 32 papers. Case reports were included, since they highlighted rare and serious complications.

Conclusion In patients $>40$ years old suspected of fish bone impaction, noncontrast $\mathrm{CT}$ is recommended and should be urgently performed, even in the presence of ambiguous symptoms. However, in patients $<40$ years old presenting within 24 hours from ingestion, imaging has little diagnostic value due to the low probability of esophageal fish bones. For this specific subgroup, in the absence of clinical findings, discharge without imaging studies may be considered safe.
\end{abstract}

\section{Introduction}

Fish bone foreign body (FFB) is one of the most common ingested foreign bodies (FBs) encountered in the emergency department (ED), accounting for 46 to $88 \%$ of the total adult FB admissions, according to some studies. ${ }^{1-12}$ It is the most common FB across Asia, the Mediterranean, and other coastal countries, assumingly due to dietary habits. ${ }^{1,7,12,13}$ Fish bone FBs are common in the pediatric population, though the estimated prevalence is lower than in adults, estimated at $\sim$ $30 \% .^{13}$ The management of patients with suspected FFB ingestion is performed by ED physicians, otolaryngologists, and gastroenterologists. Other specialists, such as cardiothoracic surgeons, may be occasionally called to assist in advanced cases or complications. ${ }^{6}$

To date, no clear algorithms are available for FFB diagnosis and management. Because of the high worldwide prevalence of FFBs on the one hand, and the severe complications potentially associated with FFBs on the other (esophageal laceration and perforation, soft tissue penetration, mediastinitis, as well as pulmonary and cardiovascular penetration), we sought to review the current information available regarding FFB management. Based on our findings, we suggest a treatment algorithm for FFB ingestion in the upper aerodigestive tract. received

February 2, 2018

accepted

July 22, 2018

published online

October 26, 2018
DOI https://doi.org/

10.1055/s-0038-1673631. ISSN 1809-9777.
Copyright (e 2019 by Thieme Revinter

Publicações Ltda, Rio de Janeiro, Brazil
License terms

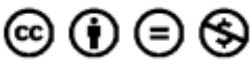




\section{Review of the Literature}

We have searched the PubMed database for the following medical subject headings (MeSH) terms: fish bone, fish foreign body AND oropharynx, hypopharynx, esophagus, flexible esophagoscopy, and rigid esophagoscopy in English, without limitations regarding the year of publication. Our search yielded 32 papers. Case reports were included, since they highlighted rare and serious complications.

\section{Clinical and Demographic Data}

\section{Age}

The age distribution of FFB impaction varies. The highest prevalence is reported in the pediatric age group (between 1-11 years old, while most patients are aged $2-4$ years old), and in middle-aged adults (30-59 years old)., ${ }^{1,2}$

\section{Impaction Site}

Fish FBs can be classified and approached subsequently, based on their site of impaction: the oropharynx, the tracheobronchial tree, and the gastroesophageal tract. The oropharynx and the esophagus (86.2\%) are more common locations than the tracheobronchial tree (13.7\%). ${ }^{1}$

Children are more prone to FB ingestion due to their tendency for environmental exploration via their mouth, to the lack of molars that decrease their ability to chew, to their inability to differentiate edible from inedible foods, and to distractions while eating. ${ }^{14}$ In this group, FFBs are most commonly located in the oropharynx, particularly in the tonsils and in the tongue base, ${ }^{13}$ due to the relative larger tonsils when compared with adults. Esophageal FFBs are rare, because the pharyngeal tonsils act as a barrier and the esophagus is narrower in comparison with adults. ${ }^{13}$

In adults, the most common impaction site is the oropharynx/hypopharynx, followed by the oral cavity and the esophagus. ${ }^{10,15,16}$ Within the oropharynx, the sites of impaction, in order of decreasing frequency, are the tonsils, the tongue base, the vallecula, and the pyriform sinus within the hypopharynx. ${ }^{2,6,13,16}$ The removal of FFBs is more difficult in the hypopharynx and is associated with higher morbidity. Predisposing factors are longer duration of the procedure due to anatomical difficulties, higher prevalence of dislodgment of the FFB during the procedure, failure of the removal of the FFB, and mucosal tears during the attempted endoscopic retrieval. ${ }^{15}$

Within the adult esophagus, the common sites are the three points of the physiological narrowing. $2,4,10,16$ Some studies report a correlation between the impaction site and the age in adult FFB impaction. Oropharyngeal FFBs appear to be more prevalent in patients $<40$ years old, while esophageal FFBs are more commonly observed in patients $>40$ years old. A possible explanation is the weakening of the swallowing mechanism, such as dysmotility of the pharyngeal muscles, epiglottic and cricopharyngeal dysfunction, and incomplete closure of the larynx, more commonly observed in older patients. ${ }^{1,2,17}$

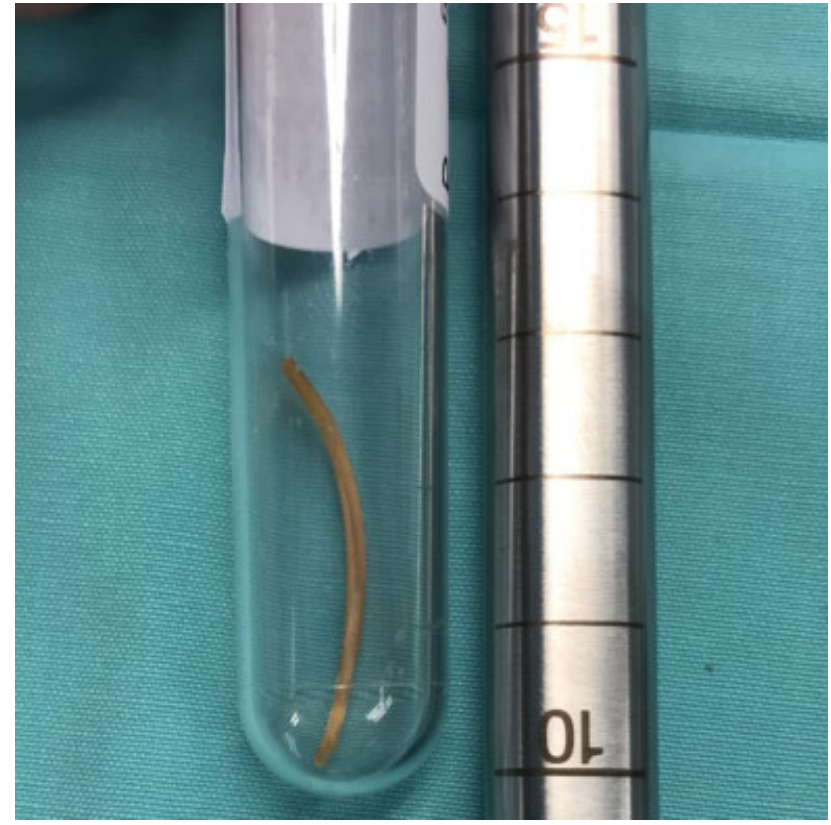

Fig. 1 A long shaped, sharp, Barbonia fish bone extracted from the proximal esophagus of a patient using a rigid endoscope.

\section{Characteristics}

The characteristics of FFBs vary according to the fish availability, the method of cooking and the eating habits. ${ }^{1,7,12}$ Most patients cannot recall and identify the type of the ingested fish. ${ }^{9}$ No specific method of cooking is considered to be a risk factor, but some studies indicated that stewed fish causes the highest number of cases of esophageal FFBs, followed by baked fish, steamed fish, and raw fish. ${ }^{1,2}$ The use of chopsticks or cutlery to eat boned fish, fish deboning in the mouth, and wearing dentures while eating are also recognized as risk factors for FFBs. ${ }^{12}$

Flat or geometric bones are more prone to esophageal impaction, whereas linear bones are more commonly impacted in the pharynx (-Fig. 1). ${ }^{1}$ Sharp, linear fish bones have a higher risk of local damage, including mucosal laceration and perforation, and penetration to adjacent tissues. ${ }^{4}$

\section{Clinical Presentation}

Symptoms vary greatly, from an asymptomatic patient to FB sensation, sore throat, dysphagia, odynophagia, retrosternal pain, blood stained saliva, and vomiting. , $^{3,11,14,15,18}$ The first symptoms can be FB sensation and localized pain. ${ }^{14}$ As time passes, localized inflammatory symptoms appear, followed by systemic symptoms, including hematemesis, fever, chest and back pain, swelling of the neck, erythema, or tenderness. Systemic symptoms in FFB impaction are rare, and may indicate pharyngeal or esophageal perforation. ${ }^{1,19}$

The value of symptoms in predicting the FFB site is controversial. The sensation of the presence of a FB is the only symptom found to correlate with the site of impaction, ${ }^{1,15}$ with a higher retrieval rate in the upper esophagus, estimated at $59 \%$, in comparison to $11 \%$ in the lower esophagus. ${ }^{1,6}$ Laterality may also be of clinical utility: in one study, 
FFB was always found ipsilaterally, whenever lateralization of symptoms was possible. ${ }^{15}$

\section{Management}

\section{Initial Management}

Airway emergency is rare, and when suspected, preventive endotracheal intubation should be considered, especially in cases of a proximal FFB, multiple FFBs, and difficulties during the removal procedure. ${ }^{3}$ In the absence of an airway emergency, history should focus on the suspected event, on the type of fish, on the time elapsed, and on the suspected site. Anatomic malformations of the esophagus, previous FB ingestion incidents and related symptoms should be questioned. $3,11,13$

An examination of the oral cavity, of the pharynx, and of the larynx, using a tongue depressor, and fiberoptic laryngoscopy is mandatory. $1,13,15,16,18$ Tenderness over the trachea may indicate the presence of FFB in the cricopharyngeal area. The absence of physical findings does not rule out the presence of FFBs. ${ }^{18}$ |The sensitivity of fiberoptic laryngoscopy in the confirmation of the presence of FFB and the identification of the impaction site is $65 \%$ in comparison to intraoperative findings, and the specificity is $100 \%{ }^{17}$

\section{Imaging Studies}

Fish FBs are difficult to identify on plain radiography, depending on their size, the bony calcification and the fish type. ${ }^{15}$ The radio-opacity of FFBs varies from species to species. ${ }^{14,16,18}$ Several studies showed that the sensitivity of plain radiography was only $32 \%$, and the false-negative rate was as high as $47 \%{ }^{1,18,20}$ The opacity level of FFBs, in a decreasing order, is as follows: bass, catfish, redfish, drum, tilapia, flounder, salmon, trout, red snapper, and tilefish. ${ }^{18}$ The lateral view is usually better for the detection of oropharyngeal and upper esophageal FFBs. ${ }^{1}$

Therefore, computerized tomography (CT) is currently the standard-of-care imaging modality in cases of suspected FFBs. $^{20,21}$ The sensitivity and specificity rates for FFB identi- fication are high in CT scans, including the relation to adjacent anatomic structures, with minimal false-negative rates. $^{2,5,16,17}$ - Fig. 2 shows an illustrative case of FFB in the esophagus. Computed tomography scans are valuable in patients presenting with suspected complications, such as soft tissue penetration, and in hypopharyngeal FFBs. ${ }^{11,22}$ Some studies suggest that ultra-low-dose neck/chest CT using the iDose 4 is an adequate first-line imaging modality for sharp esophageal FFBs before the endoscopic extraction. This imaging modality demonstrated $100 \%$ sensitivity and $97.8 \%$ specificity rates, thus allowing to keep a high image quality, while reducing the radiation dose. ${ }^{23}$ Another novel approach is the use of 64-slice multi-detector CT scan, providing twice the sensitivity in comparison to plain radiography (100 and $51.7 \%$, respectively). ${ }^{24}$

Contrast esophagography is not recommended, due to the high risk of aspiration and the diminished visualization of the esophagus if an endoscopy is attempted subsequently. However, it is beneficial in the identification of esophageal perforation, with the use of water-soluble contrast preferable to barium contrast due to its rapid absorption. ${ }^{1}$

\section{Treatment Modalities}

The immediate removal of the FFB is the preferred approach. ${ }^{11}$ According to the American Society of Gastrointestinal Endoscopy, FFB is considered an emergency, and should be removed as soon as possible, ${ }^{3,25}$ before it passes through the stomach, because between 15 and 35\% of FFBs perforate the intestines. ${ }^{5,25}$ The retrieval rate of FFBs has been found to be higher than that of other FBs, due to the higher oropharyngeal impaction prevalence, their shape and sharpness. ${ }^{6}$ Furthermore, the retrieval rate is higher within 6 hours from the event, and with symptoms at/above the cricoid level. ${ }^{6}$

Fish FBs in the oropharynx, in the tongue base, or even in the hypopharynx can be removed using a tongue depressor, an indirect laryngeal mirror, a laryngoscope and several types of forceps. ${ }^{11,13,14}$ Otherwise, the preferred treatment modality differs between flexible to rigid endoscopy. Flexible endoscopy
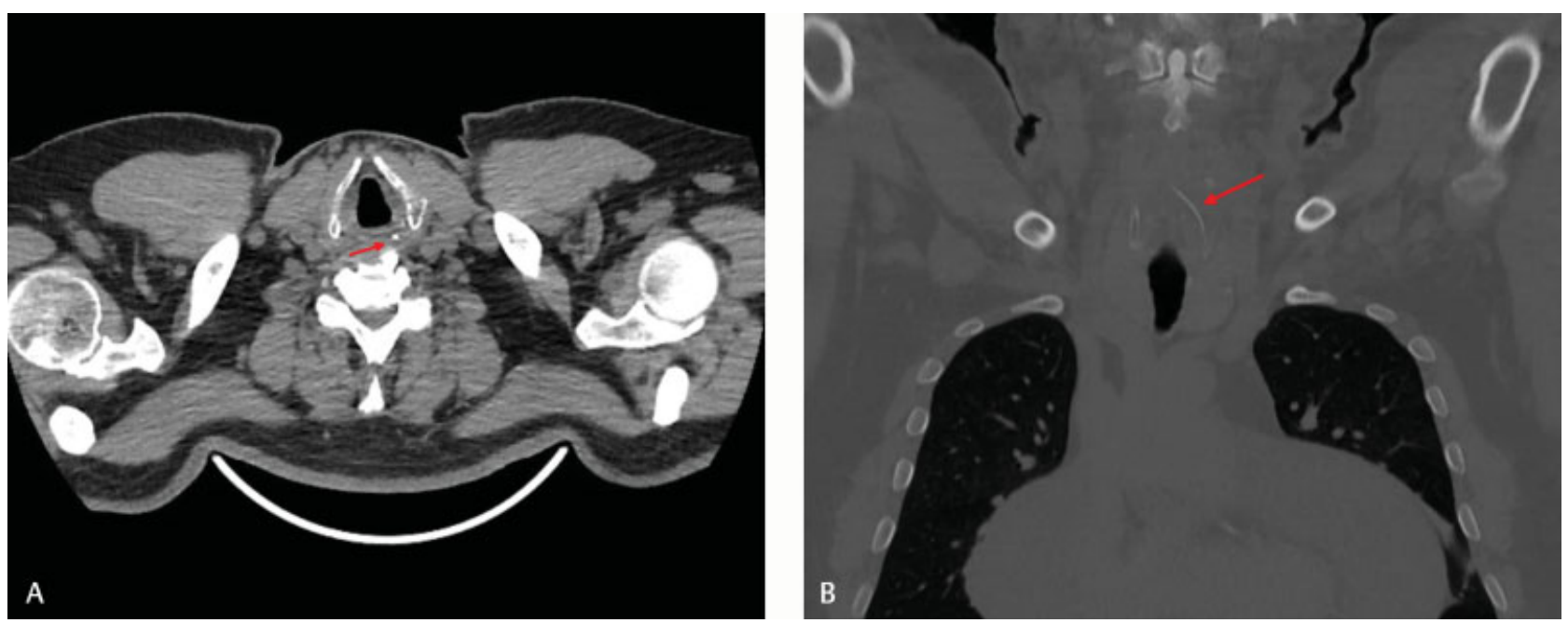

Fig. 2 Computed tomography scan without contrast showing a long, sharp, Barbonia fish bone impacted in the proximal esophagus. (A) Axial view. (B) Coronal view. 
has a low risk of perforation, a high success rate, and no need for general anesthesia, ${ }^{1,2,4,11,15,26}$ while rigid endoscopy is considered more equipped for FFB impaction in the upper esophagus or the in the hypopharynx. In these locations, the identification and retrieval of FFBs using flexible endoscopy was found to be inferior in comparison to rigid endoscopy. ${ }^{9,11,15}$ In addition, in cases of FFBs with high-risk characteristics (sharp edges, large size, FFBs impacted in high risk sites, such as the second esophageal stricture, and FFBs lodged in an angle) that are more likely to cause tissue damage, and, as well as in the presence of complications, ${ }^{6}$ rigid endoscopy might be of greater benefit. ${ }^{16}$

The use of transnasal esophagoscopy (TNE) was recently reported to be a useful tool for the removal of esophageal FFBs. ${ }^{2,16}$ The advantages of TNE are that it neither requires sedation nor general anesthesia, thus eliminating the associated risks, and it not requires fasting, which enables a quick initiation of the procedure, and is well tolerated by patients.

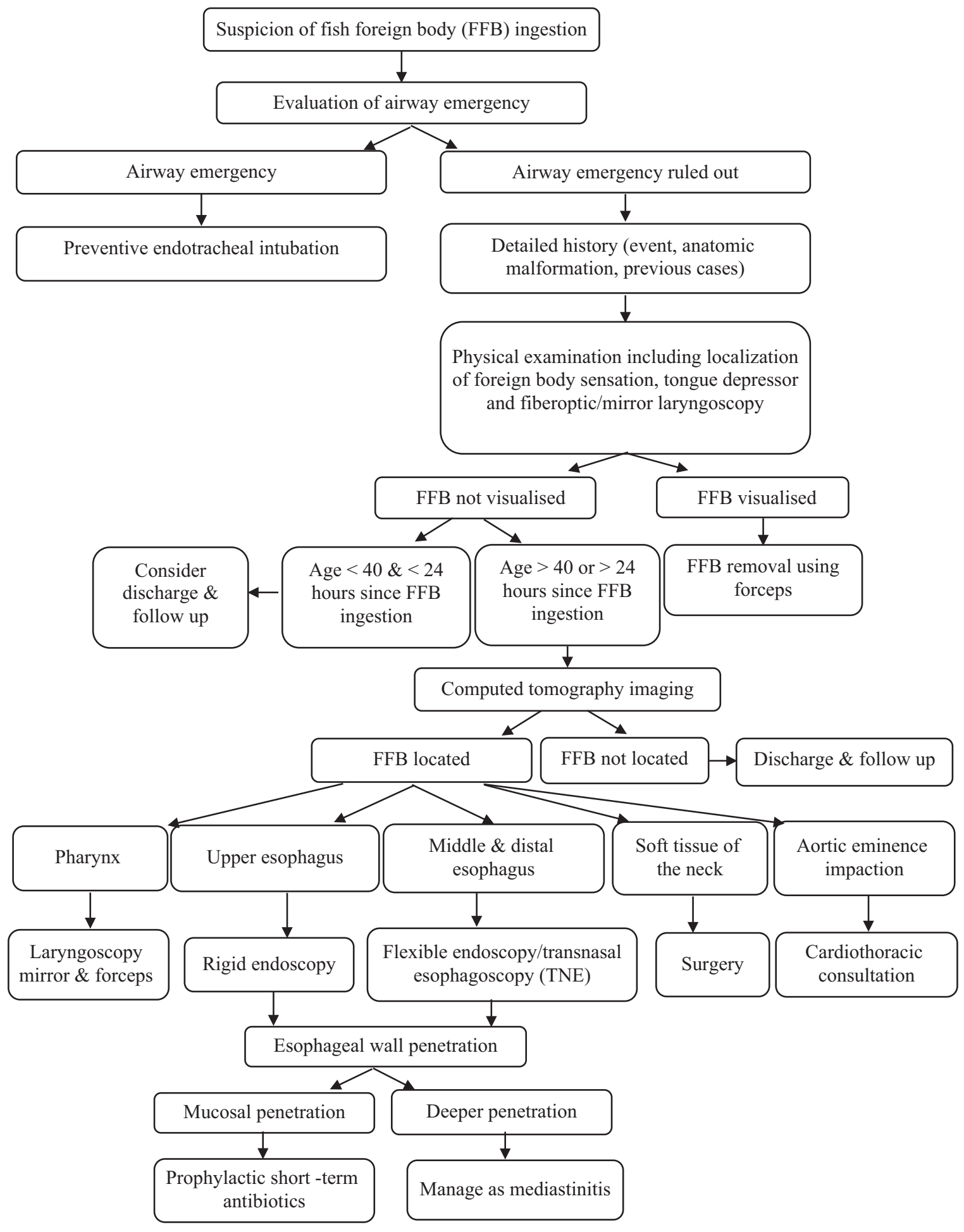

Fig. 3 Fish bone foreign body management algorithm. 
Transnasal esophagoscopy is considered less compatible for large FBs with sharp edges, but it was reported to be useful in cases of fish bone impaction without difficulty or adverse effects. ${ }^{16}$ Another option is the rigid curved laryngoscope, which has the advantage of full visualization in difficult sites, such as the hypopharynx, and allows the use of rigid forceps such as Magill forceps for retrieval. ${ }^{22}$

In cases of FFB impaction leading to surface penetration of soft tissues, a surgical approach and intraoperative imaging such as ultrasound can be incorporated. ${ }^{27,28}$ Another available option is the combination of endoscopic and surgical approaches, based on the site of impaction. ${ }^{29}$

\section{Complications}

The progression of FFB impaction can lead to serious complications, depending on the route the FFB advances. ${ }^{9}$ The main complications are laceration and penetration/perforation of the pharyngoesophageal wall, leading to infection and damage to the adjacent tissues. ${ }^{1,3}$ The risk of complications increases with a longer duration elapsed between the ingestion and the attempted retrieval ( $>24$ hours), and FFB type and length $(>3 \mathrm{~cm}){ }^{4,10,14,15}$ The greater the time elapsed until the attempted treatment, the longer the duration of hospitalization. 4

The most common complications of esophageal FFBs are laceration or ulceration, with or without minor bleeding. ${ }^{1}$ Fish FBs are reported to have the highest risk of perforation, as high as $35 \%$, in comparison to other FBs. ${ }^{1,5}$ Partial esophageal wall penetration can lead to mediastinitis and abscess formation. Complete esophageal wall penetration can cause a tracheoesophageal fistula, recurrent pneumonia, lung abscess, empyema, and pneumothorax. ${ }^{1}$ In cases of mucosal penetration, the FFB initially seals off the penetration itself, preventing significant air and liquid leakage, thus prophylactic short-term antibiotic administration is sufficient. In subsequent stages, laceration, gross perforation, and infection can develop, and the patient should be managed as having mediastinitis. ${ }^{1}$

Severe complications of FFB impaction are uncommon. Pharyngeal FFBs can cause deep neck infection, neck abscess and retropharyngeal hematoma/abscess, estimated at 3\%. ${ }^{15,30}$ Soft tissue migration of FFBs was also reported to result in esophageal dissection, penetration into the facial artery, floor of mouth hematoma, penetration of the parotid duct, and retropharyngeal abscess. ${ }^{15,29-31}$ In more severe cases, FFBs can damage the cardiovascular system. This is a relatively rare occurrence, but one with lethal repercussions. ${ }^{1}$ The FFB can advance to the heart, causing pericarditis, cardiac tamponade, infectious endocarditis, and systemic air embolism. If a sharp FFB penetrates the aortic arch eminence, a pseudoaneurysm or an aortoesophageal fistula can develop. ${ }^{1,5,32}$

\section{Discussion}

We present our management algorithm for FFB impaction, as shown in -Fig. 3.

According to Kim et $\mathrm{al}^{2}$, FFBs in younger patients $(<40$ years old) are far more likely to be impacted in the oropharyngeal region or in the upper esophagus. In children specifically, it is extremely rare to encounter FFBs in the esophagus, possibly due to differences in anatomy. Since this has not yet been established, further research is needed to determine unequivocally the causes for this age group distribution. Zhang et $\mathrm{al}^{4}$ reported that FFB is more likely to get lodged in the pharyngeal region or in the upper esophagus in the first 24 hours, and in the mid/ lower esophagus beyond that time frame. According to Marçal et $\mathrm{al}^{6}{ }^{6} \mathrm{FB}$ sensation is relatively indicative of FFB location at or above the upper esophagus.

Based on these findings, we recommend that young patients $<40$ years old, suspected of FFB ingestion in the last 24 hours, complaining of FB sensation in the upper esophagus or above, and without pathologic findings in the physical examination, can be considered for safe discharge. Nevertheless, in patients $>40$ years old or in patients presenting $>24$ hours after the suspected event, when no FFB is found, a CT scan is warranted to rule out FFB.

\section{Final Comments}

Computed tomography imaging has high sensitivity and specificity rates for the identification of FFBs and is currently considered the standard-of-care imaging modality in cases of suspected FFB impaction. However, new evidence today suggests that, in some cases, if the physical examination does not reveal signs of FFB presence, imaging exams can be discarded altogether. These findings call for further research, but if substantiated, in the future we can save many patients from unnecessary radiation.

Key Points

- Fish bone foreign body impaction is common.

- The value of symptoms in predicting the FFB site is controversial.

- Oropharyngeal FFBs appear to be more prevalent in patients $<40$ years old, while esophageal FFBs are more commonly observed in patients $>40$ years old.

- When FFB impaction is suspected, the absence of physical examination findings does not rule out the presence of FFB.

- Computed tomography is the standard-of-care imaging modality.

- Young patients with suspected recent FFB ingestion, and without findings in the physical examination can be safely discharged without imaging studies.

\section{References}

1 Kim HU. Oroesophageal Fish Bone Foreign Body. Clin Endosc 2016;49(04):318-326

2 Kim JP, Kwon OJ, Shim HS, Kim RB, Kim JH, Woo SH. Analysis of Clinical Feature and Management of Fish Bone Ingestion of Upper Gastrointestinal Tract. Clin Exp Otorhinolaryngol 2015;8(03):261-267

3 Bekkerman M, Sachdev AH, Andrade J, Twersky Y, Iqbal S. Endoscopic Management of Foreign Bodies in the Gastrointestinal Tract: A Review of the Literature. Gastroenterol Res Pract 2016; 2016:8520767

4 Zhang X, Jiang Y, Fu T, Zhang X, Li N, Tu C. Esophageal foreign bodies in adults with different durations of time from ingestion to effective treatment. J Int Med Res 2017;45(04):1386-1393 
5 Sugawa C, Ono H, Taleb M, Lucas CE. Endoscopic management of foreign bodies in the upper gastrointestinal tract: A review. World J Gastrointest Endosc 2014;6(10):475-481

6 Marçal N, Soares JB, Pereira G, Guimarães J, Gonçalves M, Godinho $\mathrm{T}$. The management of ingested foreign bodies in an Ear Nose and Throat Emergency Unit: prospective study of 204 cases. Acta Otorrinolaringol Esp 2013;64(03):197-203

7 Dagan E, Yakirevich A, Migirov L, Wolf M. The role of economics and weekend meals in impacted fish bone occurrence in central Israel. Isr Med Assoc J 2011;13(01):48-50

8 Wyllie R. Foreign bodies in the gastrointestinal tract. Curr Opin Pediatr 2006;18(05):563-564

9 Webb WA. Management of foreign bodies of the upper gastrointestinal tract: update. Gastrointest Endosc 1995;41(01):39-51

10 Damghani M, Halavati N, Motamedi N. Foreign body in the upper airway and oesophagus: a seven years study from Iran. J Pak Med Assoc 2011;61(09):859-862

11 Ashraf O. Foreign body in the esophagus: a review. Sao Paulo Med J 2006;124(06):346-349

12 Arulanandam S, Das De S, Kanagalingam J. A prospective study of epidemiological risk factors for ingestion of fish bones in Singapore. Singapore Med J 2015;56(06):329-332, quiz 333

13 Higo R, Matsumoto Y, Ichimura K, Kaga K. Foreign bodies in the aerodigestive tract in pediatric patients. Auris Nasus Larynx 2003;30(04):397-401

14 Geraci G, Sciume' C, Di Carlo G, Picciurro A, Modica G. Retrospective analysis of management of ingested foreign bodies and food impactions in emergency endoscopic setting in adults. BMC Emerg Med 2016;16(01):42

15 Ngan JH, Fok PJ, Lai EC, Branicki FJ, Wong J. A prospective study on fish bone ingestion. Experience of 358 patients. Ann Surg 1990; 211(04):459-462

16 Woo SH, Kim KH. Proposal for methods of diagnosis of fish bone foreign body in the Esophagus. Laryngoscope 2015;125(11): 2472-2475

17 Sheth N, Diner WC. Swallowing problems in the elderly. Dysphagia 1988;2(04):209-215

18 Qureshi TA, Awan MS, Hussain M, Wasif M. Effectiveness of plain $\mathrm{X}$-ray in detection of fish and chicken bone foreign body in upper aerodigestive tract. J Pak Med Assoc 2017;67(04):544-547

19 Rodríguez H, Passali GC, Gregori D, et al. Management of foreign bodies in the airway and oesophagus. Int J Pediatr Otorhinolaryngol 2012;76(Suppl 1):S84-S91
20 Ritchie T, Harvey M. The utility of plain radiography in assessment of upper aerodigestive tract fishbone impaction: an evaluation of 22 New Zealand fish species. N Z Med J 2010;123 (1313):32-37

21 Evans RM, Ahuja A, Rhys Williams S, Van Hasselt CA. The lateral neck radiograph in suspected impacted fish bones-does it have a role? Clin Radiol 1992;46(02):121-123

22 Sakaida H, Chiyonobu K, Ishinaga H, Takeuchi K. Use of a Rigid Curved Laryngoscope for Removal of a Fish Bone in the Hypopharynx. Case Rep Otolaryngol 2016;2016:9689521

23 Kim EY, Min YG, Bista AB, Park KJ, Kang DK, Sun JS. Usefulness of Ultralow-Dose (Submillisievert) Chest CT Using Iterative Reconstruction for Initial Evaluation of Sharp Fish Bone Esophageal Foreign Body. AJR Am J Roentgenol 2015;205(05):985-990

24 Park S, Choi DS, Shin HS, et al. Fish bone foreign bodies in the pharynx and upper esophagus: evaluation with 64-slice MDCT. Acta Radiol 2014;55(01):8-13

25 Ikenberry SO, Jue TL, Anderson MA, et al; ASGE Standards of Practice Committee. Management of ingested foreign bodies and food impactions. Gastrointest Endosc 2011;73(06):1085-1091

26 Gmeiner D, von Rahden BH, Meco C, Hutter J, Oberascher G, Stein HJ. Flexible versus rigid endoscopy for treatment of foreign body impaction in the esophagus. Surg Endosc 2007;21(11): 2026-2029

27 Baba S, Takizawa K, Yamada C, Monobe H. A submucosal esophageal fish bone foreign body surgically removed using intraoperative ultrasonography. Am J Otolaryngol 2014;35(02):268-270

28 Smith ME, Riffat F, Berman LH, Jani P. Intraoperative ultrasound to facilitate removal of a submucosal foreign body. J Clin Ultrasound 2014;42(09):565-568

29 Yamano Y, Uzawa K, Ito H, Tanzawa H. Endoscopically assisted removal of a fish bone penetrating the parotid duct: an unusual case. J Oral Maxillofac Surg 2014;72(07):1343-1349

30 Wei Y, Jahreiß L, Zhang Z, Albers AE. Acute airway obstruction due to retropharyngeal haematoma caused by a large fish bone in a patient with hypertension caused by a pheochromocytoma. BMJ Case Rep 2015;2015:bcr2014208644

31 Chung SM, Kim HS, Park EH. Migrating pharyngeal foreign bodies: a series of four cases of saw-toothed fish bones. Eur Arch Otorhinolaryngol 2008;265(09):1125-1129

32 Sharland MG, McCaughan BC. Perforation of the esophagus by a fish bone leading to cardiac tamponade. Ann Thorac Surg 1993;56 (04):969-971 